\title{
Pelvic Floor Exercises with Biofeedback for Stress Urinary Incontinence
}

\author{
Maria V. Capelini, Cassio L. Riccetto, Miriam Dambros, Jose T. Tamanini, Viviane \\ Herrmann, Virginia Muller
}

Division of Urology and Gynecology, State University of Campinas, UNICAMP, Sao Paulo, Brazil

\begin{abstract}
Objective: Prospective study to objectively evaluate the benefits of pelvic floor strengthening exercises associated to biofeedback for the treatment of stress urinary incontinence.

Materials and Methods: Fourteen patients diagnosed with stress urinary incontinence (SUI) were selected for this study. All patients underwent a pelvic floor training associated to biofeedback for 12 consecutive weeks. Urodynamic tests, pad test and bladder diary were analyzed at the beginning of the study, at the end and after 3 months. The King's Health Questionnaire (KHQ) was applied before and after treatment to assess the impact in the quality of life.

Results: There was a significant reduction in the pad weight (from $14.21 \mathrm{~g}$ to $1 \mathrm{~g}$ ), number of urinary leakage episodes (from 8.14 per day to 2.57 per day) and daytime frequency (from 7.93 per day to 5.85 per day). At urodynamics the authors

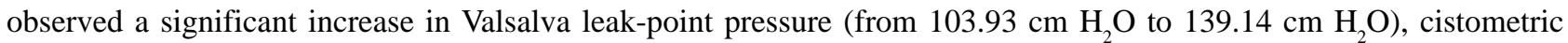
capacity (from $249.29 \mathrm{~mL}$ to $336.43 \mathrm{~mL}, \mathrm{p}=0.0015$ ) and bladder volume at first desire to void (from $145 \mathrm{~mL}$ to 215.71 $\mathrm{mL}$ ). Those differences were kept during the first 3 months of follow up. The KHQ revealed significant differences except in the case of "general health perception", which covers health in general and not exclusively urinary incontinence.

Conclusion: Treatment of SUI with pelvic floor exercises associated to biofeedback caused significant changes in the parameters analyzed, with maintenance of good results 3 months after treatment.
\end{abstract}

Key words: urinary incontinence, stress; pelvic floor; biofeedback; physical therapy modalities Int Braz J Urol. 2006; 32: 462-9

\section{INTRODUCTION}

Urinary incontinence is a common clinical complaint, particularly in post-menopausal women (1). Stress urinary incontinence (SUI) is the most common type of urinary incontinence and is defined as any involuntary leakage of urine related to any abdominal effort such as coughing or sneezing (2). It is estimated that $49 \%$ of the women with symptoms of incontinence present SUI (3). Increased life expectancy, particularly in women, has led to a higher inci- dence of urinary incontinence that keeps growing. Urinary incontinence interferes in social, physical, psychological and sexual aspects, adversely affecting self-esteem and quality of life.

Conservative treatment based on pelvic floor muscle exercises to restore the support of the pelvic organs and the urethral closing mechanism is becoming an important therapeutic option for the treatment of SUI. An additional resource is the biofeedback, which utilizes surface electromyography (EMG) for simultaneous monitoring, helping patients to identify 
the correct muscles for contraction. This method is able to detect pelvic floor muscle contractions, even when they are very weak, and simultaneously demonstrates their execution. Moreover, undesired contractions of other synergic muscle groups such as the glutei, adductors or abdominal muscles are also monitored, allowing isolation and selection of muscles of the pelvic floor.

The purpose of this study is to evaluate the effects of the treatment of SUI with pelvic floor muscle exercises using surface EMG-biofeedback through bladder diary, pad test, urodynamic studies and in the domains of the King's Health Questionnaire (KHQ).

\section{MATERIALS AND METHODS}

This was a prospective study conducted from August 2002 to December 2003. The treatment was offered to all patients with clinical history of stress urinary incontinence during medical consultation. The diagnosis of stress urinary incontinence was based on clinical history, physical examination, urodynamic study, bladder diary, pad test with standardized volume and the King's Health Questionnaire (KHQ) $(4,5)$. These measures were repeated at the end of the treatment and again three months later. The selection of patients followed the inclusion and exclusion criteria described in Table-1. For a total of 28 patients that underwent consultation during this period, $16 \mathrm{did}$ not meet the inclusion criteria and 2 refused the treatment modality due to difficulties to go to the sessions at the hospital twice weekly. All patients signed a term of informed consent. No one patient lost the followup.

The average age of the patients was 49.6 years (range from 34 to 64 years). The majority was white $(85.7 \%)$ and $57.1 \%$ had completed high school (Table2). Daily pads were used by $42.9 \%$ of the patients (mean of 3 pads per day) (Table-3). In $76.9 \%$ of the patients there has been a previous vaginal delivery and mean parity was $2.15 \pm 1.77$ ).

The patients were assisted by a multidisciplinary team consisting of physicians, nurses and physiotherapists during the treatment. They
Table 1 - Inclusion and exclusion criteria.

\begin{tabular}{l} 
Inclusion Criteria \\
Women older than 21 years of age \\
No associated neurological diseases \\
SUI due to urethral hypermobility (type I and II) \\
Exclusion Criteria \\
SUI due to intrinsic sphincteric deficiency (type III) \\
Detrusor overactivity \\
Reduced cistometric capacity and/or bladder \\
compliance \\
Patients undergoing other types of treatment for SUI \\
Cystocele, rectocele uterine prolapse of degree II \\
or higher \\
Previous surgical treatment for SUI \\
Current or recurrent vulvovaginitis \\
Current or recurrent urinary tract infections \\
\hline
\end{tabular}

SUI: stress urinary incontinence.

Table 2 - Demographic data.

\begin{tabular}{lll}
\hline Education & N & $\%$ \\
\hline High school & 8 & 57.14 \\
College & 6 & 42.86 \\
Race & & \\
$\quad$ White & 12 & 85.71 \\
$\quad$ Non-white & 2 & 14.28 \\
\hline
\end{tabular}

Table 3 - Information about pad used.

\begin{tabular}{lll}
\hline & $\mathbf{N}$ & $\mathbf{\%}$ \\
\hline $\begin{array}{l}\text { Pad used } \\
\text { Yes }\end{array}$ & & \\
No & 6 & 42.86 \\
Number of daily pads & & 57.14 \\
2 & 2 & \\
3 & 2 & 33.33 \\
4 & 2 & 33.33 \\
& & 33.33 \\
Pads type & & \\
Thin & & \\
Medium & 3 & 50 \\
Large & 2 & 33.33 \\
\hline
\end{tabular}


have undertaken 12 weeks of pelvic floor muscles exercises, monitored with surface EMG biofeedback using a protocol of standardized exercises with alternating periods of contraction and relaxation. The equipment used was the Myotrac-3 G G $^{\mathrm{TM}}$ (Thought Technology Ltd. Montreal, Canada). Adhesive surface electrodes were fixed at the abdomen to detect undesired contractions of abdominal muscles and an intravaginal electrode was placed without fixation to detect contractions of pelvic floor muscles. Sensors were connected to the abdomen and vagina with the patient in the lithotomy position. Each patient could observe the graph of the electrical activity of these muscles on a computer screen simultaneously with the exercises.

The treatment was conducted with 2 physiotherapeutic sessions of 30 to 40 minutes per week. Each session was performed at lithotomy position and consisted of 3 series of 10 sustained contractions followed by a period of relaxation. The sessions during the first 4 weeks consisted of 5 seconds of contractions followed by 10 seconds of relaxation. This series was followed by another 4 weeks of 10 seconds contractions and 10 seconds relaxations. Finally, the sessions included 20 seconds contractions followed by 20 seconds of relaxation during the last 4 weeks. The subjective parameter was the KHQ that deals with aspects of urinary incontinence, quality of life and to which extent urinary incontinence affects the life of the patients. Each KHQ domain obtains a score and therefore there is no general score. The scores range from 0 to 100 and the higher the score, the poorer the quality of life.

An increase of up to $2 \mathrm{~g}$ in the pad test was considered normal. Increases of 2 to $10 \mathrm{~g}$ were considered to be mild to moderate, 10 to $50 \mathrm{~g}$ severe and above $50 \mathrm{~g}$ very severe (6). The bladder diary included time of micturition, urinary volume, volume of fluid intake, changes of clothes, urinary leakage and activities that provoked it. The patients filled in this diary for three consecutive days. The urodynamic study was conducted according to the International Continence Society standardization and values of Valsalva leak point pressure bellow $60 \mathrm{~cm} \mathrm{H}_{2} \mathrm{O}$ were considered as diagnostic for intrinsic sphincteric deficiency.

For statistical evaluation, we utilized the Friedmann test for objective variables, the Wilconxon test for descriptive analysis, the Spearman rank correlation to verify an association between 2 variables and the generalized estimation equations to evaluate the influence of the objective variables in each KHQ domain. In all tests, $\mathrm{p}<0.005$ was considered significant.

\section{RESULTS}

Urinary leakage at the pad test before treat-

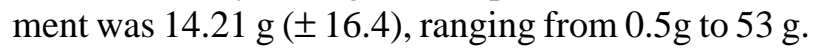
After 12 weeks of treatment, the mean urinary leakage reduced to $1.86 \mathrm{~g}( \pm 4.7)$ and $1 \mathrm{~g}( \pm 5.4)$ after 3 months. There were no significant differences between leakage either immediately or 3 months after treatment $(\mathrm{p}<0.0001)($ Table-4).

The bladder diary revealed a significant decrease in voiding frequency from 7.9 times per day to 5.8 after treatment, remaining unaltered 3 months after treatment $(p=0.0021)$. Mean of urinary leakage daily episodes also decreased from 8.1 to 0.4 post-treatment and 2.6 after 3 months (Table-5). This increase at 3 months was not significant $(\mathrm{p}<0.001)$.

There was no significant change in daily fluid intake from pre-treatment $(1,780 \mathrm{~mL})$, at the end of

Table 4-Pad test ( $g$ ).

\begin{tabular}{llllccc}
\hline & N & Mean & SD & Minimum & Median & Maximum \\
\hline Pretreatment & 14 & 14.21 & 16.38 & 0.5 & 9.5 & 53 \\
Post-treatment & 14 & 1.86 & 1.97 & 0.5 & 1 & 6.5 \\
3 months & 13 & 1 & 1.08 & 0 & 1 & 3 \\
\hline
\end{tabular}

$p<0.0001$; Friedman's test $; S D=$ standard deviation. 
Table 5 - Episodes of urinary leakage.

\begin{tabular}{llccccr}
\hline & N & Mean & SD & Minimum & Median & Maximum \\
\hline Pretreatment & 14 & 8.14 & 11.90 & 0 & 3.5 & 42 \\
Post-treatment & 14 & 0.43 & 1.09 & 0 & 0 & 4 \\
After 3 months & 14 & 2.57 & 6.20 & 0 & 0 & 22 \\
\hline
\end{tabular}

$p<0.0001 ;$ Friedman's test $; S D=$ standard deviation. .

treatment $(1,776 \mathrm{~mL})$ and at 3 months $(1,609 \mathrm{~mL})(\mathrm{p}$ $=0.6122$ ).

Mean Valsalva leak-point pressure (VLPP) was $103.9 \mathrm{~cm} \mathrm{H}_{2} \mathrm{O}( \pm 12.5)$. After the treatment, the VLPP evolved to $142.9 \mathrm{~cm} \mathrm{H}_{2} \mathrm{O}( \pm 29.2)$ and to 139.1 $\mathrm{cm} \mathrm{H}_{2} \mathrm{O}( \pm 20.7)$ at 3 months ( $<$ 0.0001) (Table-6).

Cystometric capacity was $249.3 \mathrm{~mL}( \pm 81.9)$ in the pre-treatment, with a significant increase to $367.9 \mathrm{~mL}( \pm 128.9)$ at the end of treatment and to $336.4 \mathrm{~mL}( \pm 63.2) 3$ months after treatment $(\mathrm{p}=$ $0.0015)$. Similarly, first desire to void increased from $145 \mathrm{~mL}( \pm 52.1)$ in the pre-treatment to $223.9 \mathrm{~mL}( \pm$ $73.1)$ and $215.7 \mathrm{~mL}( \pm 81.3)$ respectively after the treatment and at 3 months $(\mathrm{p}=0.0056)$.

The Spearman linear correlation coefficient was applied to verify the correlation between objective variables (VLPP and pad test) at different phases of the study. Only the immediate post-treatment phase demonstrated a linear association $(r=-0.06 ; p=$ 0.0233), which indicates that these data were inversely correlated - when the VLPP increased, the leakage decreased. However, at 3 months, no correlation could be observed $(\mathrm{p}=0.3514)$.

Analysis of 9 domains in the KHQ was performed using the Wilcoxon's paired samples test. All the domains demonstrated significant differences except for the one related to "General Health Percep- tion". All the domains presented low average scores after treatment.

The Generalized Estimation Equations were used to evaluate the statistic influence of the objective variables (pad test, VLPP, number of leakage episodes) on quality of life, in each KHQ domain, obtained during pre and post-treatment. Significant influence was observed in the following domains: physical limitations, personal relationships, emotions, sleep and moods, and degree of severity. The number of leakage episodes exclusively influenced all the domains. Valsalva leak point pressure influenced only the severity domain (Table-7).

\section{DISCUSSION}

Since Kegel attributed that slack pelvic floor muscles were a relevant factor in urinary incontinence, numerous studies proposed SUI treatment by exercises that strengthen pelvic floor muscles (7). Several studies have proved the effectiveness of strengthening the pelvic floor muscles and others have shown that these exercises in association with biofeedback are safe and effective (8).

The complementary effect of biofeedback on pelvic floor rehabilitation program is still a

Table 6 - Valsalva leak-point pressure $\left(\mathrm{cm} \mathrm{H}_{2} \mathrm{O}\right)$.

\begin{tabular}{llllccc}
\hline & N & Mean & SD & Minimum & Median & Maximum \\
\hline Pre-treatment & 14 & 103.9 & 12.54 & 90 & 101.5 & 135 \\
Post-treatment & 14 & 142.9 & 29.2 & 91 & 145 & 199 \\
After 3 months & 14 & 139.1 & 20.74 & 99 & 147.5 & 170 \\
\hline
\end{tabular}

p<0.0001; Friedman's Test. 
Table 7 - Influence of the objective variables on KHQ domains ( $p$ values).

\begin{tabular}{lllrlrrrrr}
\hline & & \multicolumn{9}{c}{ KHQ Domains } \\
& GHP * & LDL & SL & E & SM & I Im * & PL & PR & SM \\
\hline & & & & & & & & & \\
Pad test & 0.551 & 0.914 & 0.119 & 0.343 & 0.526 & 0.793 & 0.694 & 0.393 & 0.205 \\
VLPP & 0.363 & 0.063 & 0.151 & 0.387 & 0.011 & 0.215 & 0.388 & 0.173 & 0.461 \\
Leakage episodes & 0.0956 & $<0.001$ & $<0.001$ & $<0.001$ & 0.002 & 0.136 & $<0.001$ & 0.017 & $<0.001$ \\
\hline
\end{tabular}

* No significant differences in these domains. GHP = general health perception; $L D L=$ limitations of daily life; $S L=$ social limitations; $E=$ emotions; $S M=$ severity measures; I Im = incontinence impact $; P L=$ Physical Limitations; $P R=$ personal relationship; SM =sleep and mood.

controversial subject. In 1998, Berghmans et al. (9) reviewed all studies published between 1980 and 1998 that had extensive evidence of the use of biofeedback. Based on five methodologically adequate studies they concluded that there was strong evidence that biofeedback associated with pelvic floor exercises did not increase the efficacy of the treatment. On the other hand, the metaanalysis performed by Weatheral (10) led to the conclusion that biofeedback was an effective aid in strengthening pelvic floor muscles for it presented increasing cure rates. Nevertheless, these studies differ greatly regarding interventions conducted, research population, assessment measures and equipment used, making them difficult to be compared.

In the present study, we analyzed the influence of pelvic floor exercises and biofeedback on objective parameters in a prospective design. Although, we allocated a small group of patients for a nonrandomized observational study with a short followup, the other studies used retrospective analysis or lacked instruments to evaluate quality of life.

Up to date, the pad test is an important instrument in clinical assessment, quantifying urinary leakage despite great variations in behavior, appearance, poor reproducibility and accuracy, which makes it hard to compare in different studies (11). The 20 minutes pad test with a standardized infusion of $250 \mathrm{~mL}$ (6) was chosen to avoid bladder volume interference in patient's assessment. When pre and post-treatment assessments were compared, there was an improvement of $87.6 \%$. In the three-month assessment, five patients did not present any leakage during the pad test and the maximum leakage observed during this period was 3 grams, which was considered a minimum leakage related to vaginal secretion.

In another multicentric prospective study (12), 109 SUI patients were assessed to analyze the accuracy of noninvasive parameters on urinary incontinence. The 24-hour bladder diary and the pad test proved to be reliable quantification instruments of urinary leakage and number of incontinence episodes, respectively. They also concluded that increasing the tests to 48 and 72 hours increased their reliability but reduced the number of patients who were able to complete them.

We observed a reduction on urinary frequency and the number of leakage episodes in the bladder diary. Similarly, Pages (8) conducted a randomized study of 40 women with SUI treated both with and without biofeedback. A significant improvement was observed in both groups after three months of treatment. The biofeedback group demonstrated a $10 \%$ reduction in daily urinary frequency and $36 \%$ in the nocturnal frequency after four weeks of treatment. In this same group, after three months, the reduction in day and night frequencies was 5\% and 66\%, respectively. The subjective assessment in the threemonth follow up showed that $28 \%$ of patients in the incontinent group treated with exercises were cured, as opposed to $62 \%$ of the biofeedback group. Similarly, in our study, there was a reduction in both urinary frequency and the number of leakage episodes in 12 of the 14 patients who underwent treatment. At the end of the study, 10 patients had no leakage episodes, which were also maintained after three months follow up. 
In a recent study, Bo (13) found a positive correlation between increase in the maximum force of the pelvic floor muscles and decrease in the episodes of SUI. Although in our study there was a progressive increase in the surface electromyographic activity (microvolts) representing the intensity of pelvic floor muscle contraction, the authors decided not to include this parameter based on the fact that surface electromyography may suffer interferences and therefore would not be a reliable quantitative parameter. Thickness of the subcutaneous tissue, cutaneous resistance, vaginal impedance and electrodes position are some variables that undermine the value of an eventual comparison. It is also known that vaginal impedance can vary in a woman due to the menstrual cycle.

In this study, an increase was observed in the VLPP, maximum cistometric capacity and volume at first desire to void. Pajoncini et al. studied 166 women and demonstrated that maximum urethral closure pressure and VLPP measured different components of urethral function, low VLPP significantly correlating to severity of incontinence, prior urogynecological surgery and reduced urethral mobility (14). Standardized techniques regarding thickness of the catheter, bladder volume to check pressure and coexistence of genital anomalies as well as other intervenient factors should be observed (15). All measurements in our study were standardized in order to reduce variability.

The subjective parameter chosen for analysis in this study was the KHQ (4). This instrument was chosen to verify the impact of therapies on quality of life, which has been increasingly relevant and common in clinical research. International literature reveals a consensus regarding the fact that urinary incontinence can adversely affect quality of life (16) in many aspects such as psychological, physical, social, personal and sexual relationship. Robinson et al. (17) demonstrated that the impact on the quality of life of patients with complaints of urinary incontinence could be assessed using a questionnaire.

The use of this questionnaire (KHQ) (4) in patients with SUI is original, as international literature has applied this instrument to conservative management urge-incontinence. The International
Continence Society (ICS) has recommended that an assessment of quality of life should be included in all clinical studies as a complement of objective data (18). This questionnaire was chosen for our study, particularly because of its extensive approach, easy comprehension, specificity and applicability. To our knowledge, this study represents the first application of KHQ in the assessment of an urinary incontinence conservative treatment.

In this study, the treatment applied demonstrated a significant improvement in quality of life as shown by the reduced scores obtained in eight out of nine domains in KHQ. Only the "General Health Perception" domain did not demonstrate a significant difference. This expected result might undergo spontaneous changes as general health covers a wide range of aspects and it is not specifically related to urinary incontinence.

The number of leakage episodes recorded in the bladder diary was the only objective variable that significantly influenced the KHQ domains. Nevertheless, this variable was removed from the statistical analysis so that the importance of the rest of the variables could be verified. Following this trend, it was found that the VLPP significantly influenced the domain "Severity". This domain assesses the presence and intensity of the various aspects related to complaints of urinary incontinence, deals with subjects highly relevant to the incontinent patient such as daily use of pads, number of changes and the possibility of a bad smell. Therefore, a high correlation was observed between the data related to the objective and subjective variables, indicating that the assessment instruments were efficient.

In conclusion, pelvic floor exercises associated with biofeedback applied according to the described protocol promoted significant changes in the bladder diary, pad test and urodynamic parameters. Moreover, it caused significant changes in the quality of life index assessed by a validated instrument that was culturally adapted to the patient's language. The learning process offered by the biofeedback and training, followed by the maintenance of the exercises, even without supervision, may have maintained the good results observed 3 months after the supervised program of exercises was interrupted. 
These positive results must be confirmed throughout further studies randomized, with a larger number of patients and a longer follow-up.

\section{CONFLICT OF INTEREST}

None declared.

\section{REFERENCES}

1. Thom D: Variation in estimates of urinary incontinence prevalence in the community: effects of differences in definition, population characteristics, and study type. J Am Geriatr Soc. 1998; 46: 473-80.

2. Abrams P, Cardozo L, Fall M, Griffiths D, Rosier P, Ulmsten $\mathrm{U}$, et al.: The standardisation of terminology of lower urinary tract function: report from the Standardisation Sub-committee of the International Continence Society. Neurourol Urodyn. 2002; 21: 16778

3. Hampel C, Wienhold D, Benken N, Eggersmann C, Thuroff JW: Prevalence and natural history of female incontinence. Eur Urol. 1997; 32: 3-12.

4. Kelleher CJ, Cardozo LD, Khullar V, Salvatore S: A new questionnaire to assess the quality of life of urinary incontinent women. Br J Obstet Gynaecol. 1997; 104: 1374-9.

5. Weber AM, Abrams P, Brubaker L, Cundiff G, Davis G, Dmochowski RR, et al.: The standardization of terminology for researchers in female pelvic floor disorders. Int Urogynecol J Pelvic Floor Dysfunct. 2001; 12: 178-86.

6. Sand PK, Ostergard DR: Urodynamics and the evaluation of female incontinence: a practical guide. London, Springer. 1995; pp. 20-3.

7. Kegel AH: Progressive resistance exercise in the functional restoration of the perineal muscles. Am J Obstet Gyn. 1948; 56: 238-49.

8. Pages IH, Jahr S, Schaufele MK, Conradi E: Comparative analysis of biofeedback and physical therapy for treatment of urinary stress incontinence in women. Am J Phys Med Rehabil. 2001; 80: 494-502.

9. Berghmans LC, Hendriks HJ, Bo K, Hay-Smith EJ, de Bie RA, van Waalwijk van Doorn ES: Conservative treatment of stress urinary incontinence in women: a systematic review of randomized clinical trials. $\mathrm{Br} \mathrm{J}$ Urol. 1998; 82: 181-91.
10. Weatherall M: Biofeedback or pelvic floor muscle exercises for female genuine stress incontinence: a meta-analysis of trials identified in a systematic review. BJU Int. 1999; 83: 1015-6.

11. Soroka D, Drutz HP, Glazener CM, Hay-Smith EJ, Ross S: Perineal pad test in evaluating outcome of treatments for female incontinence: a systematic review. Int Urogynecol J Pelvic Floor Dysfunct. 2002; 13: $165-75$.

12. Groutz A, Blaivas JG, Rosenthal JE: A simplified urinary incontinence score for the evaluation of treatment outcomes. Neurourol Urodyn. 2000; 19: 12735.

13. Bo K: Pelvic floor muscle strength and response to pelvic floor muscle training for stress urinary incontinence. Neurourol Urodyn. 2003; 22: 654-8.

14. Pajoncini C, Costantini E, Guercini F, Porena M: Intrinsic sphincter deficiency: do the maximum urethral closure pressure and the Valsalva leak-point pressure identify different pathogenic mechanisms? Int Urogynecol J Pelvic Floor Dysfunct. 2002; 13: 30-5.

15. Payne CK, Raz S, Barbiaz JW: The Valsalva leak point pressure in the evaluation of stress urinary incontinence: technical aspects of measurement: J Urol. 1994; 151: 478.

16. .Chiverton PA, Wells TJ, Brink CA, Mayer R: Psychological factors associated with urinary incontinence. Clin Nurse Spec. 1996; 10: 229-33.

17. Robinson D, Pearce KF, Preisser JS, Dugan E, Suggs PK, Cohen SJ: Relationship between patient reports of urinary incontinence symptoms and quality of life measures. Obstet Gynecol. 1998; 91: 224-8.

18. Blaivas JG, Appell RA, Fantl JA, Leach G, McGuire EJ, Resnick NM: Definition and classification of urinary incontinence: recommendations of the Urodynamic Society. Neurourol Urodyn. 1997; 16: 149-51.

Accepted after revision:

May 10, 2006

\author{
Correspondence address: \\ Dr. Cássio L. Z. Riccetto \\ Rua Herman Muller, 429 \\ 13465-630, Americana, SP, Brazil \\ Fax: + $55193788-7481$ \\ E-mail: cassio.riccetto@uol.com.br
}




\section{EDITORIAL COMMENT}

This prospective and observational study was designed to evaluate the effect of pelvic floor muscle training with biofeedback on SUI in women. It is not clear, from the report, if women were submitted to therapeutic sessions only on the visits to the hospital, with the multidisciplinary team, or also followed home exercises, alone. The major contribution of this article was the description of results on several outcome measures, including quality of life (King's College Health Questionnaire - KHQ). Authors made considerable effort to analyze patients with a complete protocol, as suggested by the ICS, and deserve congratulations. Despite these considerations, clinical data on this trial add little to our comprehension of the role of biofeedback and muscle exercises on the treatment of women with SUI. One important question - Does biofeedback improve the results of pelvic floor muscle exercises on SUI? - cannot be clarified because study was not designed to answer it. They observed that this conservative treatment affect all outcome measures analyzed in a very favorable group of 14 women, on immediate and short term follow up. Pad tests had reduced leaks up to $0.5 \mathrm{~g}$, which is considered no leak at all. In addition, they observed reduction of incontinence episodes, as measured by voiding diaries, which affected most of all KHQ. We could wonder if this was really devoid to the treatment. We know the women who leaks little, sometimes go better even with no treatment after short or medium follow up. If we observe data on number of pads used, we see that it did not change much, suggesting that women of this population were not sure of their clinical state. Several treatment protocol including biofeedback alone, pelvic floor muscle training with or without biofeedback, electrical stimulation, magnetic stimulation and vaginal cones showed improvement in different groups of women with stress incontinence. Unfortunately, results are not consistent and did not last very long. This is in part devoid to the lack of standardization of treatment protocols. We know that pelvic floor muscle exercises, applied with a strength and intensive protocol, in women with soft leaks, have good results on the short-term follow up even without the help of biofeedback resources. We do not know how the adherence to treatment on the long term is, if the good results will remain, what is the ideal schedule for sessions and what are the role of biofeedback and electrical stimulation on these sessions and protocols. Finally, we do not know the results between different groups of women in terms of degree of severity and hormonal status. What we need in future trials addressing these questions is randomized trials, to give power and consistency to the results; analysis of different treatment protocols, to find out the ideal schedule for sessions; stratification of the results by subgroups of patients, to define people who can benefit of conservative treatment and long term follow up, to see the maintenance of the results as to, the adherence of patients. We hope that authors continue to follow their patients and augment the group treated since this will help us to answer some of these questions.

Dr. Carlos Alberto Bezerra Section of Urology, ABC Medical School Santo Andre, Sao Paulo, Brazil E-mail: carlos-a-bezerra@uol.com.br 